\title{
LA MÍMESIS COMO FILOSOFÍA DE LA RELIGIÓN
}

\author{
DESIDERIO PARRILLA MARTÍNEZ \\ Universidad Católica San Antonio de Murcia
}

\begin{abstract}
RESUMEN: Este artículo muestra la evolución de la teoría mimética como filosofía de la religión, la gran aportación de René Girard. Supone un análisis «in actu nascente» de la tesis fundamental del sistema girardiano, exponiendo sus fundamentos biográficos y académicos. Refleja, de esta manera, las influencias teóricas y los precedentes mediatos e inmediatos del principal hallazgo de su labor investigadora.
\end{abstract}

PALABRAS CLAVE: René Girard, deseo, teoría mimética, cristianismo.

\section{Mimesis as philosophy of religion}

ABSTRACT: This article shows the evolution of mimetic theory as philosophy of religion, the great contribution of René Girard. It involves an analysis «in actu nascente» about fundamental thesis of girardian system, giving the biographical and academic foundations. It reflects, in this way, the theoretical influences and precedents of his research.

KEY WORDS: René Girard, desire, mimetic theory, Christianity.

Un crítico afirmó de René Girard: «Girard es uno de los tipos puercoespines que sobreviven, según la tipología que Isaiah Berlin ha deducido sutilmente del verso de Arquíloco: La zorra sabe muchas cosas, pero el puercoespín sabe una sola cosa grande. La sola cosa grande que Girard sabe tiene un nombre: chivo expiatorio ${ }^{1}$.

En efecto, Girard encaja dentro del perfil que se atribuye al filósofo «puercoespín», si es que consideramos el deseo mimético y sus efectos victimarios como la monomanía o idea fija de su contribución investigadora.

El famoso autor de La violencia y lo sagrado nació el día de Navidad de 1924, en Avignon. Cursó estudios de Filosofía como pregrado en el Liceo de su ciudad natal, y se graduó como archivista, paleógrafo, especialista en estudios medievales de la Escuela de Chartres en París, donde estudió entre 1943 y 1947, siguiendo los pasos de su padre ${ }^{2}$.

Sus primeros intereses académicos se centraron en la vida y la historia de la cultura de sus antepasados, como se puede apreciar en su trabajo de investigación: "La vida privada en Avignon en la segunda mitad del siglo XV». Se le presentó la oportunidad de pasar un año en Estados Unidos, país al que ha estado vinculado desde 1947. Así continuó sus estudios, matriculándose en el Doctorado en Historia de la Universidad de Indiana, donde se graduó en 1950 con una tesis titulada: «American Opinion of France, 1940-1943». Aunque parezca un trabajo distante de sus concepciones sobre la mimesis, manifiesta su

1 Calasso, R., La ruina de Kasch, Barcelona, Anagrama 1983, p. 160.

2 Para esta semblanza biográfica seguimos el esquema canónico establecido por WILLiams, J., The Girard Reader, Crossroad, New York 1996, pp. 1-6. 
interés muy temprano por las formas culturales, las modas y las costumbres, pues se expresan y mueven en torno a lo que más tarde acuñará como «deseo mimético», que es el centro de su pensamiento.

Durante estos años de investigación doctoral subsistió enseñando francés y literatura, que luego será capital como fuente de su teoría y de su conversión religiosa. La literatura que enseñaba le comenzó a fascinar, hasta el punto que cuando publicó su primer libro, "Mensage romantique et verité romanesque» (1961), fue calificado como crítico literario. No obstante, en sus primeros artículos, como "El matrimonio en Avignon en la segunda mitad del siglo XV», y sobre todo "Voltaire y la historiografía clásica», se puede apreciar una fuerte investigación historiográfica que va a ser en adelante una de las peculiaridades de su tarea científica.

Como no logró publicar sus artículos a tiempo, y aunque en 1953 publicó 7 artículos, la Universidad de Indiana no lo nombró en posesión de su cátedra ${ }^{3}$. Entre 1953 y 1957 trabajó en la Universidad Duke como instructor, siendo a la vez asistente de un curso en el Bryn Mawr College. En 1957 fue nombrado profesor asociado en la Universidad John Hopkins, donde fue ascendido a profesor titular en 1961. En esta universidad encuentra a Leo Spitzer ${ }^{4}$ y a los críticos literarios de lengua francesa Georges Poulet y Jean Starobinski. Es el propio Leo Spitzer quien aconseja a Girard, tras leer el manuscrito de «Mentira romántica y verdad novelesca», la lectura del opúsculo de Max Scheler titulado «Sobre el resentimiento de la moral» ${ }^{5}$.

En este período va gestándose La violencia y lo sagrado. Entabla amistad con John Freccero que realiza su tesis doctoral ${ }^{6}$ con Charles Singleton ${ }^{7}$, un especialista de Dante que procede de Harvard. Girard está en la universidad John Hopkins como profesor asociado, pero es precisamente Charles Singleton quien a raíz de

3 Lagarde, F., René Girard on la christianisation des sciences humaines, Peter Lang, New York 1994, p. 191.

4 Leo Spitzer (1887-1961), crítico literario y filólogo austríaco, considerado, junto con Charles Bally, como uno de los fundadores de la estilística moderna. Concluyó una dilatada carrera de profesor en la universidad Johns Hopkins. Sus libros Stil-studien (1928) y Romanische Stil und Literaturstudien (1931) recogen la mayor parte de sus ensayos. Publicó asimismo Essays on Historical Semantics (1948).

5 El editor Stefano Tomelleri ha reunido todos los ensayos de René Girard acerca de la idea teórica de "resentimiento». Cfr. GIRARD, R., Il risentimiento. Lo scacco del desiderio nell'uomo contemporáneo, Cortina, Milan 1999.

6 Freccero, J., The Neutral Angels from Dante to Matteo Palmieri, Baltimore, tesis doctoral, Johns Hopkins University 1958.

7 Charles S. Singlenton es uno de los más grandes especialistas de Dante del siglo xx. Enseñó tanto en Harvard como en John Hopkins. Publicó renombradas monografías que son obras de referencia en materia de literatura italiana. En esta fase hizo una edición de Decamerón de Boccacio (Baltimore, The Johns Hopkins University Press, 1974), pero sobresalen sus estudios sobre Dante: Dante's Work: An Essay on the Vita Nuova (1949); Dante's Commedia: Elements of Structure (1954), y Journey to Beatrice (1957), todos publicados por Harvard UP. Publicó además una traducción anotada de la Divina comedia en seis volúmenes para la Bollingen Series of Princeton University Press, 1970-1975. 
la publicación de «Mentira romántica, verdad novelesca» le promociona a profesor. La amistad de John Freccero le inicia en la obra de Dante, donde Girard encuentra un ámbito de aplicación para la teoría mimética ${ }^{8}$. Imparte los cursos de tercer ciclo a alumnos tan dotados como Eugenio Donato, Eric Gans y Andrew McKenna, que luego serán tan relevantes para el desarrollo de la hipótesis del deseo mimético'. A su vez entra en contacto con Cesareo Bandera, un especialista en Cervantes y Calderón, que pasa a ser uno de los investigadores más afines a su pensamiento ${ }^{10}$ y con quien coincidirá en la Universidad de Buffalo.

Este fue un período crítico que produjo sus primeras obras relevantes, pero cuya base fue un proceso de cambio interior. En algunas entrevistas ha contado que en otoño de 1958 trabajaba en "Mentira romántica y verdad novelesca» desde la perspectiva propia de la «desmitificación», según la manera intelectual de la época.

En este trabajo revisionista descubrió la mimesis como gran arma destructora, capaz de demoler aquello que los modernos hemos considerado como más propio: nuestros deseos. Pero esta experiencia de «desmitificación» radical estaba muy cerca de la experiencia que la tradición cristiana ha llamado «metanoia» o «epistrofé», es decir, "conversión».

Las primeras ideas sobre una obra siempre eran diferentes de la versión final. El primer borrador era una autojustificación; podía ser al elegir al antagonista, quien será un chivo expiatorio del autor, el rival cuya maldad debe quedar demostrada al final de la obra; podía ser al construir al héroe, con quien se identifica el escritor, y quien será reivindicado a lo largo de la obra. El buen escritor reconocerá que una obra escrita así no vale nada, pues se basa en una distinción entre lo bueno y lo malo carente de autorreflexión. Entonces, el novelista se convierte en marioneta de sus propios demonios. Así, un gran novelista se hace capaz de describir la maldad tanto de los otros como de sí mismo, mientras antes era un trabajo ilusorio de inculpación. Esta experiencia rompe el orgullo y la vanidad del escritor; se trata de un verdadero quiebre existencial ${ }^{11}$.

8 Resultado de esta aportación es una sistematización más profunda del deseo mimético, como se advierte en: "From "The Divine Comedy" to the Sociology of the Nobel», Sociology of Literature and Drama, Elizabeth Burns y Tom Burns (ed), Penguin Books 1973, pp. 101-108.

9 Eric Gans es profesor de francés en UCLA. Estudió con René Girard en Johns Hopkins a finales de los años 1960. Partiendo de la tesis girardina del «mecanismo del chivo expiatorio» formula la teoría de la antropología generativa en libros como The End of Culture: Towards a Generative Anthropology, Berkeley, University, University of California Press 1985; Originary Thinking: Elementes of Generative Anthopology, Stanford University Press 1993. Es editor de la publicación on-line Antropoetics, dedicados a los debates de la antropología generativa: http://www.anthropoetics.ucla.edu. Andrew J. MacKenna es el editor de Contagion: Journal of Violence, Mimesis and Culture, la publicación más comprometida con el desarrollo de la hipótesis del deseo mimético.

10 Bandera, C., The Sacred Game, University Park, PA, Pennsylvania State University Press 1994.

11 Girard, R., The Girard Reader. James Williams (ed), New York, Crossroad, pp. 283-284. Cfr. GIRARD, R., Les origines de la culture, Desclée de Brouwer, 2004, pp. 23-60. 
Su conversión intelectual consistió en obtener la vivencia de la «verdad novelesca», en contra de toda forma de ideología, incluyendo la lectura romántica, según la cual las relaciones interpersonales son espontáneas. Pero en la Pascua de 1962 este proceso culminó en una conversión al cristianismo que le permitió liberarse del mimetismo violento que le dominaba y poder diferenciar así el amor perfecto respecto de las relaciones de celos, envidia y odios impotentes, por tanto, de los conflictos y de la violencia de la que somos culpables en nuestra vida cotidiana. La vivencia de conversión supuso para Girard un vaciamiento de la perversión mimética, de la rivalidad ciclotímica y el autoengrandecimiento que implica, así como un envío poderoso a comunicar a otras personas esta experiencia ${ }^{12}$.

Es en esa época, finales de los 50, cuando experimenta el momento más decisivo de su vida. Padece un cáncer de piel. Investigando las tradiciones religiosas de las culturas primitivas se da cuenta de que todas tienen un común denominador: el fenómeno del sacrificio ritual. La Iglesia católica no es una excepción. Durante la cuaresma de 1959 investiga la tradición católica como hasta ahora había investigado las tradiciones de las tribus amazónicas, como una tribu más entre tantas; pero encuentra una diferencia crucial entre la celebración pascual de Cristo y todas las demás tradiciones humanas referentes al sacrificio. Decide celebrar la cuaresma «como si» fuera un cristiano más, al modo del ficcionalismo de Vaihinger.

Este tiempo coincide con el trabajo de escritura y reflexión de su primer libro ya citado. Le descubre el cristianismo desde una perspectiva en la que nunca había reparado: la defensa y rehabilitación de las víctimas de la violencia que cada comunidad considera sagrada. Junto a los descubrimientos que le depara la escritura del último capítulo del libro, Girard padece una revelación y se convierte al catolicismo.

«Provengo de una familia típica del catolicismo occidental [dice a Carlos Mendoza en una entrevista concedida a raíz de la aparición de su penúltimo libro en el 2010] ${ }^{13}$ : una madre piadosa, en cierto modo conservadora, marcada por las costumbres de sus ancestros, incluida la simpatía por la tradición de la realeza católica e incluso con algunos tintes de racismo. Mi padre, por el contrario, pertenecía a la tradición radical socialista cercana al ateísmo. En mi juventud me alejé de la Iglesia y regresé a ella justo antes del Concilio Vaticano II, pero ahora, buscando vincularme con la larga tradición cristiana, y dadas mis tendencias estéticas y antropológicas fue la Iglesia antigua la que me llamó la atención. Por estos motivos, por ejemplo en Stanford, me gusta participar en una Misa gregoriana, pues el canto pleno preserva con gran vigor ese dinamismo místico propio de la fe. Al mismo tiempo debo decir que eso no

12 GiRARD, R., Cuando empiecen a suceder estas cosas... Conversaciones con Michel Treguer. Madrid. Encuentro 1997, pp. 150-153. Schwager, R., Conversión and authenticy: Lonergan and Girard, COV\&R Meeting 2000, Boston College, 31 mai-3 juni 2000.

13 Mendoza, C., Pensar la esperanza como apocalipsis. Conversación con René Girard, abril de 2008. [http://www.letraslibres.com/index.php?art=12884]. 
significa que me sitúo del lado de una teología conservadora. Cristo es quien ha mostrado el fracaso de la religión arcaica, sacrificial, desmontando sus mecanismos victimarios y llamando a la humanidad a romper el círculo de la violencia mimética. Creo que por eso su mensaje es universal y trascendente».

Pero esta confesión reciente es fruto de una evolución y parte de una inequívoca juventud descreída e indiferente a la vida de la fe. Girard ha tenido una evolución realmente interesante. Desde el punto de vista de una biografía espiritual, este hombre singular, denominado por J.-M. Domenach, el «Hegel del cristianismo» ${ }^{14}$, está todavía escribiendo su historia, que no pasa de ser la de un hombre corriente, pero con visos de revolucionar el pensamiento y los conceptos que los hombres tienen de sí mismos. Su historia es la de un escéptico intelectual que, de pronto, se encuentra ante la palmaria presencia de una intuición sobre lo que el hombre es, que le abre una ventana a un horizonte comprensivo amplio y profundo.

En la entrevista que concede a J.-M. Treguer relata con sencillez los acontecimientos más importantes de su historia personal: «Todo se me presentó en 1959. Sentía que había allí un bloque en el cual he ido penetrando poco a poco. Estaba enteramente allí, en el punto de partida, todo junto. Por eso no tengo ninguna duda... No hay "sistema Girard". Exploto una intuición única pero muy densa».

J.-M. Treguer, que pretende aviesamente desenmascararle como un topo cristiano disfrazado de ilustrado, nos dice: «Ya ha contado usted cómo, después de una juventud moderadamente cristiana, llegó, primero por los novelistas, por Proust, a sus ideas actuales. Personalmente sospecho que disimula un acontecimiento que usted nunca habría contado, una experiencia mística, un verdadero encuentro con Dios al estilo del "camino de Damasco"».

A lo que responde nuestro autor:

«Decir que mi juventud fue cristiana, incluso moderadamente, es una exageración. Mi madre, por supuesto, era una excelente católica, a la vez que sólidamente creyente y de espíritu abierto. Cuando digo esto a los devotos del psicoanálisis menean la cabeza con aire de entendidos en la materia. Eso les tranquiliza enormemente. Pero hay algunos que no se contentan con el "retorno a la madre" Ciertas damas que palpan mi complejo de Edipo lo encuentran "durius-culo", por no decir duro. He soportado ya tres o cuatro artículos sobre este tema. Parece al respecto que no soy muy de fiar.

No disimulo mi biografía, pero no quiero caer en el narcisismo al que todos estamos inclinados. Tiene razón, por supuesto, hay una experiencia personal detrás de lo que digo. Comenzó hace treinta y cinco años. En el otoño de 1958 trabajaba sobre mi libro acerca de la novela, en el decimosegundo y último capítulo que se titula "Conclusión». Reflexionaba sobre las analogías entre la experiencia religiosa y la del novelista que se descubre embustero sistemático, embustero en beneficio de su "yo», el cual no se constituye en el fondo más que

14 Cfr. Domenach, J.-M., Las ideas contemporáneas, Barcelona, Kairos 1983. 
de mil mentiras lentamente acumuladas, capitalizadas, a veces, durante toda una vida. Terminé por comprender que estaba a punto de vivir una experiencia del tipo aquel que yo describía. Embrionario entre los novelistas, el simbolismo religioso en mi caso se pone a funcionar completamente sólo, y a incendiarse espontáneamente. No podía hacerme ilusiones sobre lo que me sucedía, y estaba totalmente desconcertado pues tiraba firmemente de mi escepticismo. Me veía muy mal yendo a la Iglesia, arrodillándome, etc. Estaba, además, en otro espíritu, repleto de lo que los viejos catecismos llamaban el «respeto humano».

Intelectualmente estaba convertido, pero permanecía incapaz de poner mi vida de acuerdo con mis pensamientos. Durante un período de algunos meses, la fe se convirtió para mí en un goce delicado que rechazaba los otros placeres, una golosina más en una vida que no tenía nada de criminal, cierto, pero que estaba hecha de self-indulgence.

Como mi conversión me había hecho sensible a la música, escuchaba mucha. De esta época data la poca cultura musical que poseo, en particular en lo que se refiere a la ópera. Las bodas de Fígaro son para mí, cosa curiosa, la música mística por excelencia. Junto con el canto gregoriano. Me he dedicado a gustar también toda una música «moderna» que no había apreciado jamás anteriormente: Mahler, Stravinski, los rusos contemporáneos.

Durante el invierno del 59 enseñaba ya en John Hopkins, pero daba un curso en Bryn Mawr College donde había pasado cuatro años, y hacía la ida y vuelta Baltimore-Filadelfia cada semana en los viejos vagones chirriantes y bamboleantes del Pennsylvania Railroad. Como paisaje, contemplaba sobre todo la chatarra y los terrenos baldíos de esa vieja región industrial, Delaware y el Sur de Filadelfia, pero mi estado mental lo transfiguraba todo y, a la vuelta, el menor rayo de sol poniente suscitaba en mí verdaderos éxtasis. Fue en ese tren, una buena mañana, en el que me descubrí, justo en medio de la frente, un pequeño grano que no quería cerrarse, uno de esos pequeños cánceres de piel que, en verdad, son muy poco peligrosos; pero el médico al que consulté se olvidó de informarme de ello, en razón, pienso, de la extrema inquietud que él había concebido después de haberme mirado por encima y escuchado algunos instantes, con la idea de que pudiera atravesar el Atlántico en cualquier momento sin retribuirle sus honorarios... Felizmente, tenía seguros médicos, y todo lo que debía hacerse se hizo para librarme para siempre de mi pequeño grano. (...) Y he aquí que, poco después, se declaran unos efectos un tanto anormales justo la derecha de la minúscula operación. La serenidad de mi médico se vio un poco turbada, mucho menos, a decir verdad, que la primera vez, mientras que la mía, al contrario, lo fue mucho más. Me pareció claro que mi cáncer conocía un nuevo desarrollo, que esta vez podía ser fatal.

Mi dermatólogo estaba serio, y, desde esa época, él simboliza a mis ojos todo eso que hay de formidable y fatal al mismo tiempo en la medicina americana, tal vez la mejor del mundo, pero también bastante implacable, no sólo en el sentido financiero sino por su extremo cuidado en no tranquilizar a la clientela, en no nutrirla de ilusiones mentirosas. Esa medicina me recuerda un poco esos bandidos de los caminos que, mientras le vacían a uno los bolsillos a toda velocidad, 
le amenazan de muerte continuamente. Es inútil oponerles la menor resistencia. Algunos instantes más tarde se encuentra uno en el suelo sano y salvo.

En lo que a mí concierne, el período de angustia duró un poco más de tiempo. Comenzó en la semana septuagésima. Antes de las reformas litúrgicas del último Concilio, el domingo abría la septuagésima, un período de dos semanas consagrado a la preparación de los cuarenta días de cuaresma, durante el cual los fieles, a imitación de Jesús y de sus cuarenta días de ayuno en el desierto, se supone que hacían penitencia in cinere et cilicio, "con ceniza y cilicio».

Fue una excelente preparación de cuaresma la que yo hice ese año, se lo aseguro, y la cuaresma que siguió fue excelente también, pues mis preocupaciones se acrecentaron hasta el punto de privarme del sueño, hasta el día en que, tan de repente como habían empezado, se resolvieron mediante una última visita a mi oráculo médico. Habiendo hecho todos los análisis necesarios, el excelente hombre me declara curado, el miércoles santo precisamente, es decir, el día que, en la semana santa, precede a la Pasión propiamente dicha y a la fiesta de Pascua, conclusión oficial de toda penitencia.

No he conocido jamás una fiesta comparable a esa liberación. Me veía muerto y, de golpe, había resucitado. Lo más maravilloso para mí en ese asunto es que mi convicción intelectual y espiritual, mi verdadera conversión, se había producido antes de mi gran mieditis de cuaresma. Si se hubiera producido después, nunca hubiera creído verdaderamente. Mi escepticismo natural me habría persuadido de que la fe era el resultado del canguelo que tenía. El canguelo no podía tener como resultado la fe. La duración de mi noche oscura coincidió, con exacta precisión, con el período prescrito por la Iglesia para la penitencia de los pecadores, con tres días de gracia, los más importantes de todos, misericordiosamente disminuidos, sin duda para que yo pudiera reconciliarme con toda quietud con la Iglesia antes de la fiesta de Pascua.

Dios me había llamado al orden con un punto de humor, bien merecido en el fondo por la mediocridad de mi caso. En los días que siguieron a la Pascua, consagrados litúrgicamente al bautismo de los catecúmenos, hice bautizar a mis dos hijos y me casé católicamente. Estoy persuadido de que Dios envía a los hombres cantidad de signos que tienen alguna existencia objetiva para los prudentes y los sabios. Aquellos que no ven estos signos los tienen por imaginarios, pero aquellos a los cuales son destinados no pueden equivocarse en ello, pues viven la experiencia desde dentro. Enseguida comprendí que, si la superaba, el recuerdo de esa prueba, me sostendría durante toda mi vida, y es justo eso lo que ha sucedido».

Los intelectuales que comprenden la importancia de sus descubrimientos, pero que siguen anclados en la corriente anticristiana, quieren limpiarle de esa contaminación con la ortodoxia. Lo intentan una y otra vez en artículos, comentarios, y en las entrevistas a las que le someten, pero Girard no deja lugar a la ambigüedad, si bien es verdad que él mismo fue en tiempos prisionero de esa ambigüedad. En este sentido continúa su declaración a Michel Treguer:

«Desde el principio, mi cristianismo se ha bañado en una atmósfera de tradición litúrgica. Hay gente muy bien intencionada para conmigo, y 
convencionalmente anticristianos, que quieren a toda costa hacer de mí, para defender mi reputación en medios intelectuales, un herético de tomo y lomo, un enemigo feroz del "cristianismo histórico" dispuesto a poner bombas en todas las pilas de agua bendita.

¿Diciendo que "la Iglesia ha permanecido durante largo tiempo sacrificial", he añadido verdaderamente mi patada ritual a la de todos los asnos que persiguen salvajemente a la Santa Madre en el momento actual? Sin duda he demostrado, es necesario confesarlo, cierta demagogia mimética en la expresión. Hubiera hecho mejor situando mi propuesta en nuestra historia religiosa total. Pero no quería repetir el error de los fariseos de los que hablaba hace un momento, aquellos que dicen: "Si nosotros hubiéramos vivido en tiempos de nuestros padres, no habríamos participado con ellos en la muerte fundadora". No quisiera, sin embargo, condenar la fidelidad, la obediencia, la paciencia, la modestia de los cristianos ordinarios y de las generaciones que nos han precedido. Todas esas virtudes nos hacen falta tremendamente. Pertenezco demasiado a mi época para poseerlas yo mismo, pero las venero. Nada me parece más conformista, por el contrario, nada me parece más servil en el momento actual, que la mitología trasnochada de la "revolución".

Restos de jactancia vanguardista salpican mis obras, pero mis verdaderos lectores cristianos no quedan confundidos por eso, el padre Schwager, el padre Lóhfink, el último von Balthasar, el padre Corbin, el padre Alison, y algunos otros» $»^{15}$.

Posteriormente, fue director del departamento de Lenguas Romances en la Universidad John Hopkins entre 1965 y 1968. En este período facilitó un Simposio que fue importante para el surgimiento de la teoría crítica en Norteamérica. Junto con Eugenio Donato y Richard Macksey organizó la conferencia internacional «Los lenguajes de la crítica y las Ciencias del Hombre» ${ }^{16}$, en el que participaron Roland Barthés, Jacques Derrida, Jean Hyppolite, Jacques Lacan, Jean-Pierre Vernant, entre otros. Levi-Strauss, que estaba invitado, anuló su viaje y no asistió al Simposio. Girard consultó a Michel Deguy para realizar la sustitución y éste le sugirió que invitase a Jacques Derrida puesto que se disponía a publicar dos ensayos capitales sobre la escritura ${ }^{17}$. La participación de Derrida con su ponencia: "La structure, le signe et le jue dans le discours des sciences humaines» ${ }^{18}$, y su posterior ensayo "La pharmacie de Platon», le mostró que debía contar con este crítico. Posteriormente Girard desarrollará el concepto de «suplemento» y de «Phármakos» como víctima expiatoria desde

15 GIRARD, R., Cuando estas cosas comiencen a suceder, Encuentro, Madrid 1997, p. 195.

16 R. Macksey y E. Donato (eds), The Languages of Criticism and the Sciences of Man, Baltimore, The Johns Hopkins University Press 1970, pp. 246-265. René Girard escribió la introducción «Tiresias and the Critic», pp. 15-21.

17 Efectivamente, De la grammatologie es publicado un año después del coloquio: DerRIDA, J., De la grammatologie, Paris, Minuit 1967.

18 Derrida, J., Structure, sign, and play in the discourse of human sciences, en Richard Macksey y Eugenio Donato (eds), op. cit., pp. 246-265. 
el análisis de Derrida sobre la escritura medicina/veneno para la memoria, sacando los análisis del campo del lenguaje y la textualidad y llevándolos al campo extratextual de la actualidad social y a la historia ${ }^{19}$.

El estudio sobre el Phármakos le llevó a comenzar a estudiar las religiones primitivas a partir del concepto de deseo mimético, con lo que comprendió que la mimesis suele llevar a la violencia colectiva contra una víctima, en lo que Girard ha llamado el mecanismo del «chivo expiatorio». Eugenio Donato le aconseja entonces leer los antropólogos ingleses donde encontraría innumerables ejemplos del deseo mimético. Frazer supuso una iluminación, y fue su época de mayor intensidad creativa. Pasa de un antropólogo a otro sin interrupción (Frazer, Tyler, Robertson-Smith, Radcliffe-Brown, Bronislaw Malinowski, etc.). Entre 1965 y 1968 desarrolla su investigación del mecanismo fundador aplicado a la etnología, ahondando en monografías sobre culturas primitivas. Antes de esta inmersión en la antropología Girard se interesa entre 1963 y 1964 por la tragedia griega, especialmente, en Edipo donde detecta el deseo mimético ${ }^{20}$. El estudio comparativo de los mitos y las tragedias griegas, junto con las crónicas medievales y los textos de persecución, le permiten concretar cada vez más la hipótesis del mecanismo del chivo expiatorio y su evolución histórica. Además, queda fascinado por la comprensión de Freud en Tótem y Tabú, según la cual todos los mitos tienen un origen violento, aunque Freud no llegó a captar el mecanismo fundador. Todo esto quedó plasmado en La violencia y lo sagrado (1972), que fue publicada al comienzo de su período como profesor distinguido de la Universidad del Estado de Nueva York en Buffalo, donde permaneció hasta 1976. Precisamente en esta universidad comienza a interesarse por Shakespeare, para Girard el teórico del mimetismo por excelencia.

René Girard conoció la sociología de masas de Gabriel Tarde en Stanford en una antología de sus escritos en inglés ${ }^{21}$, antes de entrar en contacto con Émile Durkheim. Leyó Las formas elementales de la vida religiosa justo después de haber escrito La violencia y lo sagrado Para Girard, G. tarde y G. Le Bon han efectuado un trabajo ejemplar pero banal sobre la idea de imitación. Abordan la imitación bajo diversos planos de consciencia, y explican así las relaciones culturales a partir de ella. Han tenido intuiciones remarcables, pero secundarias porque no han descubierto la rivalidad mimética y sus consecuencias. Han recogido un conjunto de observaciones curiosas, pero de importancia limitada,

19 McKenna, A., Violence and difference, Girard, Derrida and Deconstruction. Chicago, University of Illinois Press 1992. Cfr. GIRARD, R., "Origins: A view from literature», en F. J. Varela y J.-P. Dupuy (ed), Understanding Origins of Life, Mind and Society, Dordrecht-BostonLondres, Kluwer Academic Publisher 1992, pp. 27-42.

20 Edipo y las Bacantes de Eurípides jugaron un papel esencial en la idea de linchamiento fundador. Vid. GIRARD, R., De l'experience romanesque au mythe Oedipien, "Critique» 22 (1965), pp. 899-924. Cfr. Symétrie et dissymétrie dans le mythe d'Oedipe, "Critique» 24, (1968), pp. 99-135. Une analyse d'Oedipe Roi, en Critique sociologique et critique psychanalytique, Institut de Sociologie, université libre de Bruxelles 1970, pp. 127-163.

21 Tarde, G., On Communication and Social Influence. Selected Papers, The University of Chicago Press 1969. 
sin finalizar jamás en una teoría satisfactoria. Quedan prisioneros de la definición tradicional, aristotélica, donde a la mimesis se le amputa su componente de violencia recíproca y se la considera una fuerza únicamente pacífica. Al margen de estas carencias, Girard considera infinitamente superior la aportación de Durkheim. Sin embargo, nuestro autor no le debe a Durkheim su educación antropológica sino que, como hemos visto, se instruyó en la etnología inglesa clásica (1850-1950) en particular Radcliffe-Brown y su libro Estructura y función en la sociedad primitiva. La obra de Malinowski ocupó también un lugar prioritario, pese a que la noción de «transcendencia social» («transcendencia desviada» en Girard) procede de Durkheim.

De Lévi-Strauss asimila en esta época la noción de diferenciación binaria, pero la incluye en una perspectiva diacrónica, y es de todos los autores mencionados el que resulta más interesante para la investigación sobre la paradoja. A diferencia de Lévi-Strauss, para el cual todo pensamiento consiste en pasar de la cantidad continua a la cantidad discreta, Girard busca conciliar los dos tipos de pensamiento, pues una teoría completa tiene necesidad de uno y de otro. Girard desde muy temprano ${ }^{22}$ advierte la importancia de estas bipolaridades descubiertas por el estructuralismo pero propone la paradoja mimética a fin de enmendar sus deficiencias: una teoría verdadera tiene necesidad de estabilidad sincrónica, pero también de dinamismo diacrónico. Interesa un sistema donde se considere la estructura y el devenir de la estructura. La diferenciación binaria ha sido aplicada más sistemáticamente por Lévi-Strauss que por Durkheim, demasiado sistemáticamente hasta el punto que el estructuralismo desaloja el componente histórico de su investigación. Sin embargo presupone que la estructura diferencial y bipolar es universal, y no hay identidad porque el lenguaje no puede conciliar los contrarios ni decir lo idéntico de ambos.

La paradoja en Girard pretende superar este escollo dejado por la aportación estructuralista: la paradoja permite superar las limitaciones de su método. El estructuralismo no puede trabajar con las continuidades, ni sobre procesos que van de la indiferenciación a la diferenciación, o del desorden al orden o recíprocamente. Con la paradoja del deseo y su evolución paradójica se asumen las aportaciones del programa estructuralista y se subsanan sus deficiencias. En la conclusión de El hombre desnudo (el volumen IV de Mitológicas) Lévi-Strauss condena el sacrificio ritual privándole de toda función e incluso de significación y los mitos diferenciados se relegan al ámbito de lo estético y lo lúdico dado que no nos dicen nada que nos instruya sobre el orden del mundo, la naturaleza de lo real, el origen del hombre y su destino. Para Girard, mito y rito son los dos

22 GIRARD, R., Disorder and order in mythology, en Disorder and Order, op. cit., pp. 80ss. Para Claude Lévi-Strauss, la indiferenciación y el desorden están presentes en la mitología únicamente para formar un contraste lingüístico, una estructura binaria y contradictoria. Para Girard esta bipolaridad manifiesta los dos elementos indispensables para suscitar la crisis que es preludio para el mecanismo del chivo expiatorio. Para la crítica pormenorizada a Lévi-Strauss ver: GIRARD, R., Literatura, mimesis y antropología, Gedisa, Barcelona 1997, pp. 161-201. 
pasos de la indiferenciación a la diferenciación. La aproximación puramente lingüístico-estructuralista nos vuelve incapaces de imaginar que la mitología pueda explicar la indiferenciación. La paradoja mimética asume la bipolaridad estructuralista pero la revisa y complementa con el factor temporal-diacrónico, devolviendo la inteligibilidad que el estructuralismo desde su perspectiva exclusivamente sincrónica había negado a los fenómenos culturales-simbólicos donde se manifiestan estas bipolaridades omnipresentes ${ }^{23}$.

La traducción al inglés de La violencia y lo sagrado en 1977 origina dos debates potentes ${ }^{24}$, que serán determinantes en la popularización de la teoría mimética dentro de la comunidad científica y en el desarrollo de la colaboración multidisciplinar respecto a dicha teoría. Eric Gans, uno de los colaboradores más estrechos de Girard, critica a nuestro autor que sólo haya dedicado el capítulo VI al deseo mimético ${ }^{25}$. Sin embargo, Girard ya había empezado a plantearse un tratamiento en profundidad sobre el tema, integrando bajo su dinamismo todos los fenómenos culturales. Girard advierte que no puede proseguir su investigación sin pronunciarse acerca del deseo mimético y sus principios. Se propone una sistematización de esta tesis básica a fin de sofisticar su aplicación en vista de las perspectivas interdisciplinares que están abriendo los nuevos colaboradores.

En 1976 aceptó la cátedra John M. Beall en la Universidad John Hopkins. En 1978 la Universidad le publicó «To double business bound. Essays on Literature, Mimesis and Anthropology». En este contexto aparece su libro más importante, Des choses cachées depuis la fondation du monde (1978), donde urgido por esta necesidad de tematizar de forma metódica el fenómeno mimético del deseo, entabla diálogo con los psiquiatras Jean-Michel Oughourlian y Guy Lefort. Este libro está dividido en tres secciones: 1) Antropología Fundamental, 2) Las Escrituras Judeo-Cristianas, 3) Psicología sistémica o interindividual. En este libro comienza a percibir con mayor hondura que en La violencia y lo sagrado el carácter paradójico del deseo a partir de las observaciones de Bateson sobre el «Double Bind». Es también la primera vez que Girard se declara cristiano y pone en primer plano la mediación externa como única forma de mimesis pacífica y adaptativa, teniendo a Cristo como único intermediario de esa relación mimética.

Desde 1981 Girard aceptó el cargo de profesor de Lengua, Literatura y Civilización Francesa, en la Cátedra Andrew B. Hammond de la Universidad de Stanford, de la cual se retiró en 1995. En esta universidad será donde se publique la revista Anthropoetics, dirigida por el lingüista Eric Gans e inspirada en la obra de Girard. De esta época son sus libros: Le Bouc émissaire (1982), La Route

23 Scubla, L., Lire Lévi-Strauss: Le déploiement d'une intuition, Paris, Editions Odile Jacob, 1998.

24 Uno en el área francófona, retratada en la revista Esprit, noviembre de 1973, p. 513581. Y en el ámbito de lengua inglesa, Diacritics, 8, 1 (primtemps, 1978).

25 Gans, E., Signs of Paradox: Irony, Resentment, and Other Mimetic Structures, Stanford, Stanford University Press 1997. 
antique des hommes pervers (1985), Shakespeare: le feux de l'énvie (1990), Quand ces choses commenceront... Entretiens avec michel Treguer (1994). En 1996, James Williams edita The Girard Reader, obra que sintetiza sus posiciones fundamentales.

De este período hay que destacar la creación del Colloquium on Violence and Religion (COV\&R), en 1990, que se propone explorar, criticar y desarrollar el modelo mimético de las relaciones entre la violencia y la religión en la génesis y el mantenimiento de la cultura. Este Coloquio publica The Bulletin of the Colloquium on Violence and Religion, que sale cada dos años, desde la Universidad de Innsbruck, y la Revista anual Contagion: Journal of Violence, Mimesis and Culture (desde 1994) ${ }^{26}$, publicada por la Universidad Loyola en Chicago.

A partir de 1995, su actividad se ha centrado en la resolución paradójica del deseo mimético en la Persona de Cristo. Sus últimas publicaciones son Je vois Satan tomber comme l'eclair (1999), Celui par qui le scandale arrive (2001). En estas obras se puede apreciar el trabajo sobre un aspecto que Girard no ha considerado aún terminado: el mimetismo pacífico que surge ante la Presencia de Cristo, la novedad total del acontecimiento cristiano y las relaciones entre el Evangelio y la mitología. Su intención es divulgativa y apologética. Pretende mostrar cómo el Evangelio permite leer y descifrar los mitos y, en consecuencia, los sistemas y mecanismos de la violencia mimética. En 2002 se publicó La voix méconnue de réel. Une théorie des mythes archaïques et modernes, que es una recopilación de artículos aparecidos antes en inglés en To double business bound y en The Girard Reader. En 2004 apareció su última obra Les origines de la culture ${ }^{27}$. Desde el 2005 forma parte de los «cuarenta inmortales» de la República francesa. Su obra ha sido traducida a varias decenas de lenguas hasta este momento.

Sus discípulos más próximos han percibido esta evolución hacia el factor paradójico y han realizado una labor de sistematización muy técnica, vinculando la filosofía girardiana con los paradigmas de la «autoorganización», la «física del caos» o el «azar organizado»" ${ }^{28}$.

Donald Kennedy, el presidente de la Universidad de Stanford, estaba a cargo del área de ciencias humanas cuando Girard abordaba el grueso de su investigación. Kennedy desarrolló una intensa labor de investigación multidisciplinar, acogiendo a varios premios Nobel como Kenneth Arrow, Iliya Prigogine y Johannes Salks. En 1986 la universidad nombra a René Girard, junto a

26 William, J., The Girard Reader. New York, Crossroad-Herder 1996, p. 6.

27 Otras obras que pueden introducirnos en la vida y obra de nuestro autor son: WeBB, E., Philosophers of Consciousness. Polany, Lonergan, Voegelin, Ricoeur, Girard, Kierkegaard, Seattle and London 1988. Lundager Jensen, H. J., René Girard, Frederiksberg 1991. Fleming, C., Rene Girard: Violence and Mimesis, Cambridge, Polity Press 2004. KIRWAN, M., Discovering Girard. London, Darton, Longman \& Todd 2004. Bureau, R., Le Oeuvre de René Girard, en: «Archives de Sciences Sociales des Religions» 54/2 (1982), pp. 127-137. CASINI, F., Bibliographie des études girardiennes en France et en Italie, Paris, L'Harmattan, 2004. M. Bertrand, René Girard: un mythe anti-moderne en: «Archives de Sciences Sociales des Religions» 54/2 (1982), pp. 137-148.

28 Orsini, A. C., La pensée de René Girard, Ed. Retz, París, 1986, p. 163. 
Jean-Pierre Dupuy, codirector del «Program for Interdisciplinary Research». Organizaron tres coloquios en los cuales colaboraron estrechamente. En septiembre de 1987, «Understanding origins»; en mayo de 1988, «Paradoxes of self-reference in the humanities. Law and the social sciences»; y, en octubre de 1988, «Vengeance: A colloquium in literature, philosophy and anthropology» ${ }^{29}$. Ya en 1981 se celebró el Coloquio de Cerisy-La-Salle con el título "L’Auto-organisation de la physique au politique ${ }^{30}$. En el mismo año, Girard organiza en la Universidad Stanford el coloquio «Désordres et Ordres»" ${ }^{31}$, que dará lugar a dos institutos de investigación: Centre de Recherches en Epistémologie et Autonomie (CREA) ${ }^{32}$, en la Escuela Politécnica de París, y el Laboratorio de Dinámica de Redes (LDR). El CREA fue fundado en 1982 junto con Jean-Marie Domenach bajo las sugerencias inspiradas por la obra de Jean Ullmo. Las investigaciones del CREA en la década de los ' 80 han sido determinantes en la epistemología de Girard y su tratamiento de la paradoja mimética ${ }^{33}$. Desde entonces la paradoja mimética queda establecida como un tópico recurrente en sus libros. JeanPierre Dupuy resume la contribución de Girard en los siguientes términos:

«Aprés une très longue éclipse, l'importance de ce paradoxe dans tout les domaines de la vie et de la pensée a été brusquement reconnue au XX siècle: Russell et Gödel le découvraient dans les fondements des mathématiques, Gregory Bateson à l'origine de la schizophrénie, George Spencer-Brown au fondement de toute connaissance, René Girard au coeur même du monde humain, à la source du sacré et des cultures» ${ }^{34}$.

En los artículos anteriores a la década de los 80 y en «Mentira romántica verdad novelesca», Girard expone su teoría del deseo mimético en una clave neohegeliana, muy en la línea del clima académico que se respiraba desde los años 50 bajo el influjo del revival de Alexander Kojève. Empleaba expresiones y giros sacados del idealismo de Fichte (Yo y No-yo, este último interpretado como Tú)

29 Las actas del primer coloquio están editadas en: Francisco Varela y J.-P. Dupuy (ed), Understanding Origins: contemporary views on the origins of Life, Society, Dordrecht, Boston, Kluwer Academic Publishers 1992.

30 P. Dumouchel y J.-P. Dupuy (ed), Colloque de Cerisy. L'auto-organisation: De la physique au politique, Seuil, París 1983.

31 P. Livingston (ed), Disoder and order, Anima Libri, Stanford University Press, Stanford 1984. Prigogine, I., "Order out of chaos», o. c., pp. 41-60; ARrow, K., «The economy as order and disorder», o. c., pp. 162-176; von Foester, H., «Disorder/Order: Discovery or invention?», o. c., pp. 177-189.

${ }_{32}$ GiRARD, R., La ruta antigua de los hombres perversos, Anagrama 1990, p. 77. Ibidem, Cuando estas cosas comiencen a suceder, Encuentro, Madrid 1994, pp. 79-80.

33 Cfr. Atlan, H. y Dupuy, J.-P., Mimesis and Social Morphogenesis: Violence and the Sacred from a System Analysis Viewpoint, en G. E. Lasker (ed), Applied systems and Cybernetics, vol III, Pergamon Press, New York 1981. J.-P. Dupuy y Michel Deguy (ed), René Girard et le Problème du mal, Grasset, París 1982. Dumouchel, P., Violence et vérité, Grasset, París 1985. Dupuy, J.-P., Ordres et Désordres, Seuil, París 1990. Dupuy, J.-P. y Teubner, G., Paradoxes of Self-reference in the humanities, Law and the social sciences, "Stanford Literature Review», VII, 1-2, 1990.

34 Dupuy, J.-P., Ordres et désordres, Seuil, 1990, p. 120. 
o del personalismo dialogal. Utilizaba la terminología acuñada por Sartre para su análisis fenomenológico-existencial de la convivencia intersubjetiva, así como todo cuanto podía ofrecerle la filosofía de la subjetividad y la proliferación de la "psicología profunda» o "psicología de la consciencia» en sus diversas escuelas (estructuralista, freudomarxista, etc.); finalmente, reducía la noción de paradoja al esquema dialéctico básico del «deseo de reconocimiento» hegeliano. El hecho de que sus primeros análisis miméticos se centraran sobre obras literarias tradicionalmente interpretadas en clave romántica, simbolista o meramente formal, propició aún más esta confusión, tanto más cuanto Girard nunca realiza exposiciones sistemáticas del sistema mimético sino que lo da por supuesto a lo largo de la aplicación que de él hace sobre los fenómenos culturales que estudia, ya sean novelas como al principio o, posteriormente: períodos históricos, fenómenos persecutorios o religiosos, etc. Como el mismo Girard reconoce, esta limitación teórica provocó ciertos equívocos respecto de la comprensión del mimetismo ${ }^{35}$ que era asimilado a una nueva interpretación del «deseo hegeliano de reconocimiento» o a un mero criterio esteticista para la interpretación textual literaria, sin relación alguna con la realidad extratextual.

Como Girard no contaba con un instrumental conceptual adecuado para investigar y tematizar la paradoja de la mimesis tenía que recurrir a los elementos que la cultura ambiente ponía a su disposición. La mimesis, por tanto, quedaba sepultada por los presupuestos implícitos en estas teorías, de manera que impedían captar la originalidad de dicho mecanismo. Girard en ocasiones ha reconocido que el trabajo de escribir supone para él una labor agotadora y ardua; la incapacidad de expresar o simplemente entender desde los parámetros habituales la novedad del sistema mimético tal vez sea una buena explicación de esta dificultad insalvable. Paralelamente, pone de manifiesto la aportación que supuso la teoría cibernética para Girard a través de este contacto con Jean-Pierre Dupuy. Sólo mediante la teoría cibernética de sistemas recursivos circulares estamos en condiciones de realizar una adecuada «conversión al fantasma» del sistema mimético. De este modo, la conceptualización de la mimesis encuentra en la cibernética su expresión idónea, así como el único método capaz de sostener la progresiva adecuación de esta labor investigadora.

Jean-Pierre Dupuy inició sus investigaciones en el CEREBE (Centro de Investigación sobre el Bienestar) de París y en el CIDOC (Centro Intercultural de Documentación) de Cuernavaca, Méjico. El CEREBE fue fundado por Philippe d'Iribarne, futuro girardiano, que resumió los trabajos de la asociación en dos obras: La Politique du bonheur (París, Ed. Seuil 1973) y Le Gaspille et le Decir (París, Fayard 1975).

Ivan Illich ${ }^{36}$ puede considerarse la cabeza visible de este movimiento intelectual vinculado a la autogestión de los años 70-80 que pretendía investigar los

35 Entrevista a René Girard, Diacritics 8 (1978), pp. 31-54.

36 Ivan Illich (Viena, 4 de septiembre, 1926 - Bremen, 2 de diciembre, 2002), pensador polifacético y polémico. Estudió Histología y Cristalografía en la universidad de Florencia. Entre 1932 y 1946 estudió Teología y Filosofía en la Universidad Pontificia Gregoriana del 
fundamentos socioculturales del fenómeno de la «consumación ostentatoria»

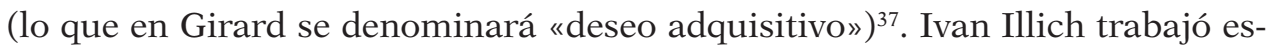
pecialmente en la noción de autonomía y su relación con la heteronomía como se puede apreciar sobre todo en el capítulo III, «Les deux dimensions de la contre-productivité institutionelle», de su libro Némesis médicale. L'expropiation de la santé (Le Seuil, 1975) ${ }^{38}$, donde formula su teoría de la "contraproductividad», es decir, un método especulativo que sirve para detectar y dar cuenta de las paradojas, o efectos contraintuitivos, que toda sociedad humana alberga en su seno y que Illich hace derivar de los fenómenos de heteronomía-autonomía en dichas sociedades. «Contraproductividad», en puridad, significa producción del contrasentido paradójico. Estas paradojas surgen en las relaciones interindividuales por el hecho de que cada individuo acusa en sí mismo un doble imperativo: ser Uno mismo (autónomo), pero al precio de serlo todo para Otro (heterónomo). El individualismo de las sociedades occidentales encumbra a valores supremos la autonomía, la originalidad y la singularidad. Se pretende que el yo sea autónomo y su voluntad espontánea. Se opone así el deseo según el Otro con el deseo según Uno mismo. Esta ideología oculta el hecho de que el sujeto saca del Otro sus deseos, en un movimiento tan fundamental y original que lo confunden con la voluntad de ser Uno mismo.

«Tal es la paradoja del yo humano, la unidad misteriosa, en cualquier hombre, de la autonomía y de la heteronomía más radicales. Ambas pulsiones nos arrastran en direcciones encontradas y jamás pueden llegar a ser complementarias, pero son para siempre inseparables, pues se alimentan mutuamente y su emparejamiento une a los hombres entre sí de manera inextricable, al mismo tiempo que los divide, entre sí y en su propio interior. Ésta es la verdadera fuente de los conflictos que enfrentan a los hombres los unos a los otros a la vez que consigo mismos. Cuanto más quiere el hombre volverse divinamente autónomo, más abandona concretamente a sus semejantes al modesto grado de autonomía de que podría disfrutar, y más se entrega, atado de pies y manos, a innumerables tiranos ${ }^{39}$.

Vaticano, y trabajó como párroco en Nueva York. En 1956 fue nombrado vice-rector de la
Universidad Católica de Puerto Rico, y en 1961 fundó el Centro Intercultural de Documen-
tación (CIDOC) en Cuernavaca Mexico, un centro de investigación que impartía cursos a
los misioneros de Norteamérica. Tras 10 años, las posturas del CIDOC le enfrentaron con el
Vaticano, y en 1976 el centro se cerró con el consentimiento de los que lo componían. A partir
de los 80, Illich viajó repartiendo su tiempo entre los Estados Unidos de América, México y
Alemania. Hizo una estancia como Profesor visitante de Filosofía y de «Ciencia, Tecnología
y Sociedad» en la Universidad Estatal de Penn, también impartió clases en la Universidad de
Bremen.
37 Dupuy, J. P., Detour and Sacrifice: Illich and Girard, en: The Challenges of Ivan Illich: A
Collective Reflection, Lee Hoinacki and Carl Mitcham (ed), New York: State University of New
York Press 2002, pp. 189204 .
38 ILlich, I. D., Némesis médica, Barral, Barcelona 1975.
39 GIRARD, R., Shakespeare. Los fuegos de la envidia, Anagrama, Barcelona 2004, p. 189. 
En enero de 1976 el CIDOC celebró un seminario organizado por Valentine Borremans, donde la reflexión del grupo investigador avanzó hacia la cibernética y la teoría de redes o sistemas. La influencia de Heinz von Foester, Francisco Varela y Henri Atlan ${ }^{40}$ fue decisiva para la formalización de la autonomía en las teorías del hombre, mediante la tematización de los llamados «sistemas autónomos» con conceptos tales como "sistemas autoorganizados», "Runaway» o el vínculo entre los «efectos de signo contrario» desarrollados en secuencia a partir del acto de apropiación que serán tan determinantes en la obra del propio Girard.

La teoría de los «efectos de signo contrario» o, simplemente, «efectos de signo", dependen del deseo de apropiación, y nos permiten esclarecer las paradojas de las sociedades industriales. Dicha teoría reposa sobre el postulado de la autonomía como principio fundamental del hombre, aunque esta autonomía repose y acabe manifestándose en un contrasentido precisamente porque la autonomía reposa sobre la heteronomía. La obra más acabada de este período es La traición de la opulencia de J.-P. Dupuy y J. Robert (ed. Gedisa, 1979).

Los efectos significativos de la consumación engendran una dinámica paradójica, un "efecto de composición», puesto que se conjugan o componen elementos contradictorios provocando la perplejidad intelectual. Dupuy entiende por «consumo ostentatorio» exactamente lo mismo que Girard por «deseo adquisitivo». Tales son al menos algunas de las conclusiones de los más de 10 años de búsquedas llevadas en el CEREBE sobre el lugar de la economía en las sociedades industriales. Sus análisis sobre los «efectos significativos» de la consumación en las relaciones interpersonales han conocido un cierto éxito en la literatura «autogestionaria».

A medida que avanzaba la investigación sobre la paradoja uno de los colaboradores comenzó a destacar sobre los demás. A él le debemos una mejor comprensión de las afirmaciones girardianas. Es el caso de Jean-Pierre Dupuy, autor que desde la Universidad de Stanford ejerció una influencia considerable en el desarrollo de la teoría mimética. Tanto es así que desde La violencia y lo sagrado, su primera obra sistemática sobre el mimetismo, toda la producción de Girard ha tenido por protagonista el tema del deseo como paradoja, haciéndose eco incluso de la noción de "partage», una de las aportaciones básicas de Dupuy y sus colaboradores (André Orléan, Lucien Scubla, etc.).

$40 \quad$ Henri Atlan, nacido el 27 de diciembre de 1931 en Blida, Argelia, es de tradición Talmúdica y doctor en medicina (1958, París). Doctor en Ciencias Políticas (1973, París-VII). Asociado al Centro de Búsquedas de la NASA (California, 1966-68) y profesor invitado del Instituto Weizmann de Israel (1970-1973). Comenzó en biología celular para continuar en epistemología y en filosofía. Es uno de los pioneros de las teorías de la complejidad y de la autoorganización del ser vivo. AtLan, H., L'organisation biologique et la théorie de l'information, Hermann, Paris 1972. Entre le cristal et la fumée: essai sur l'organisme vivant, Seuil, París 1979. Morin, E., El orden y el desorden, en: El Método 1. La naturaleza de la naturaleza, Seuil, Paris 1977, pp. 33-93. 
La noción de «partage» es la primera formulación de lo que en Girard pasará a llamarse "mimetismo paradójico» y el método que Girard usará para su estudio de los fenómenos culturales, refinando la intuición contenida de modo embrionario en el capítulo VII («Freud y el complejo de Edipo») de $\mathrm{La}$ violencia y lo sagrado, convirtiéndose en presupuesto fundamental de toda su producción posterior. «Partager» en Dupuy (y «paradójico» en Girard) es tanto la acción de descomponer algo en sus partes, partirlo, cuanto la acción de compartir, tomar parte o participar en algo ${ }^{41}$; supone pues la unión de dos cosas inmediatamente contradictorias, separar y unir, hacer partes o partición y formar parte o participar; en el primer caso algo se descompone en los elementos que lo forman, para hacerlos claros y distintos; en el segundo, lo que está separado es medido para establecer un puente que los una, que haga copartícipes aquellas dos partes que de otra manera permanecerían como elementos incompatibles. Observada desde esta intrincada semántica, la obra de Girard puede ser descrita como un trabajo de "partage». De modo que a nuestro modo de ver resulta imposible entender el método girardiano sin el método de Dupuy. Ambos son sistemas simbióticos. Sin embargo, como Dupuy declara en Ordres et desórdres (1982, p. 260) este método de análisis mediante rastreo y explicitación de paradojas tiene como precedente a Ton Siebers o James Gleick con la «Física del caos»".

Sorprende la coherencia con que Girard reconoce la existencia de un método superior al «partage». Más aún, este método superior es el camino de apertura a la realidad en todos sus factores. Según Girard, es además el que introdujo en la historia el aparato conceptual necesario para formular el «partage». Este método, origen de cualquier otro método, lleva siglos operante entre nosotros sin que nadie (o casi nadie) se haya dado cuenta de la labor que ha estado llevando a cabo. Es la revelación cristiana y el anuncio del Reino de los Cielos a través de la Iglesia.

De ahí que dedique la última parte de su obra al fenómeno de la revelación evangélica, que supondría algo así como el «partage» de todo otro "partage». Según Girard, la revelación cristiana es la que ha hecho posible la revelación de la contención y, en consecuencia, de toda la paradoja subyacente a la cultura humana. Sin embargo, el cristianismo no es un caso de contención. Aunque es un hecho histórico y, por tanto, social, es un caso único en la historia pues implica dentro de la sociedad una lógica extrasocial (extraña, ajena, a la lógica contenida en toda sociedad humana pasada, presente y venidera). El cristianismo introduce el deseo mimético, de forma paradójica pero real, en una nueva dinámica de funcionamiento, inaugurando una nueva forma de vínculo interindividual (o, como afirma Girard «interdividual») que origina una masa

41 J.-P. Dupuy desarrolla y aplica explícitamente este método (implícito en toda su obra anterior) en El pánico, Gedisa, Barcelona 1999, y en El Sacrificio y la envidia. El liberalismo frente a la justicia social, Gedisa, Barcelona 1998.

42 Gleick, J., Chaos: Macking a science, Penguin Book, Nueva York, 1988. 
social mimetizada y movida por el amor y no bajo los imperativos de la rivalidad mimética.

Por eso el cristianismo no puede ser estudiado desde el «partage», o método de contención. Pues contener supone la acción de refrenar o someter a un límite algo que, en caso contrario, quedaría suelto y se desbordaría, pero también denota la situación de llevar o tener dentro de sí algo que de este modo deja de ser considerado como exterior; que algo contenga alguna otra cosa significa que la tiene a raya y la incorpora. El deseo mimético contiene en este doble sentido la violencia; por ello, sirve de criterio de juicio para el método de "partage». Como el cristianismo no contiene violencia mimética ninguna, el "partage» no se puede aplicar al acontecimiento cristiano. Más bien la revelación cristiana permite mostrar cómo surgió a través de la Pasión, muerte y Resurrección de Jesucristo, el espacio necesario para empezar a formular el «partage».

Esto es lo que Girard ha tratado de exponer a lo largo de su producción desde el Misterio de nuestro mundo. Pero esto tal vez sólo lo entienda un cristiano. Girard lo entiende y por eso lo consignamos. En todo caso, permítasenos romper una lanza en favor del carácter eminentemente filosófico de esta afirmación girardiana. Girard no sostiene que la realidad sea así porque es cristiano. Más bien es cristiano porque juzga que la realidad es así, dado que para Girard la fe es un juicio razonable acerca de lo real. De manera que retomamos un tema tan querido para la tradición filosófica como es la relación «Fe-razón», pero planteado en unos términos novedosos y originales ${ }^{43}$.

Universidad Católica San Antonio de Murcia (UCAM)

Desiderio Parrilla Martínez dparrilla@ucam.edu

[Artículo aprobado para publicación en noviembre de 2013]

43 «Los evangelios son promovidos por una inteligencia que no es la de los discípulos y en la que veo claro que está más allá de lo que usted, yo, todos nosotros, podemos concebir sin ellos, una razón de tal manera superior a la nuestra, que después de dos mil años le descubrimos nuevos aspectos. Se trata de un proceso que nos supera, ya que no hemos podido concebirla por nosotros mismos; y, por tanto, o somos capaces de asimilarla, o lo seremos pronto. Es, pues, perfectamente racional pero de una razón más alta que la nuestra. Se trata aquí, según mi punto de vista, de una iluminación nueva de una grandísima idea tradicional: la razón y la fe se sostienen mutuamente. Fidens quaerens intellectum, y viceversa. He aquí un razonamiento tomista, pienso, pero aplicado a un campo, la antropología, que, en la época de Santo Tomás, no existía en el sentido del mundo moderno. Y, que es la cuestión una vez más, es esa Luz que es a la vez lo que debe ser visto y lo que permite ver, Deum de deum, Lumen de Lumine». Treger, J.-M., Cuando estas cosas comiencen a suceder... Entrevistas con Michel Treguer, Encuentro, Madrid 2002, p. 153. Cfr. GIRARD, R., Veo a Satán caer como el relámpago, Anagrama, 2002. Llano, A., Deseo, violencia, sacrificio, Eunsa, Pamplona 2004. von Balthasar, H. URS, Teodramática, volumen IV, Encuentro, Madrid 1992, pp. 273-289. 